\title{
Neuropsychiatric Symptoms as the first Manifestation of Systemic Lupus Erythematosus in a Male - A Case Report
}

\author{
Authors \\ Dr Rakesh Mohanty ${ }^{1^{*}}$, Dr Tanuja Mohapatra ${ }^{2}$ \\ ${ }^{1}$ Junior Resident Department of General Medicine VSS Institute of Medical Science and Research \\ Burla - 768017 \\ ${ }^{2}$ Junior Resident Department of Paediatrics SCB Medical College and Hospital Cuttack \\ *Corresponding Author \\ Dr Rakesh Mohanty
}

\begin{abstract}
Systemic lupus erythematosus (SLE) is an autoimmune multisysytem disorder with myriad presentation. This often results in a delay in diagnosis and appropriate treatment of the condition. There are limited reports on the neuropsychiatric findings as the first manifestation of systemic lupus erythematosus in male patients. Herein, we report a case of a male patient who presented with a five days history of behavioural problems and was later diagnosed to have systemic lupus erythematosus.
\end{abstract}

\section{Introduction}

Systemic lupus erythematosus (SLE) is a multisystem inflammatory disorder affecting multiple organ systems including the nervous system $^{(1)}$. Nervous system is affected in more than half of all patients during course of the disease ${ }^{(2,3)}$. SLE is significantly more prevalent in females than in males, with a female- to- male ratio of $11: 1^{(4)}$. However findings of neuropsychiatric symptoms as first manifestation in male patients subsequently diagnosed to have systemic lupus erythematosus is rare. Here we report a case of a male patient who presented with behavioural problems and was later diagnosed to have Neuropsychiatric systemic lupus erythematosus.

\section{Case Presentation}

A 26 year old male presented to our department of general medicine outdoor with history of abnormal behaviour for 5 days. He had no history of fever, any trauma or recent vaccination. He was not a diagnosed case of any seizure disorder. His bladder and bowel habits were normal. On further history taking it was revealed that he had on and off multiple joint pain, arthralgia and photosensitivity since last few years. His physical examination revealed he had a butterfly shaped rash in the malar area sparing the nasolabial folds and an oral ulcer. His blood pressure was 120/80 mmhm and pulse rate was $84 /$ min regular. His body temperature was $98.6 \mathrm{f}$. With history and examination a diagnosis of neuropsychiatric systemic lupus erythematosus was suspected. Subsequently ANA and DsDNA titres were sent along with routine investigations and MRI scan of brain was planned.

Laboratory investigations revealed high titres of ANA and DsDNA which were positive for 
systemic lupus erythematosus. His other blood investigations were in normal range. He was negative for HIV, HBV, HCV infections. MRI brain revealed no significant abnormality.

Subsequently a diagnosis of neuropsychiatric systemic lupus erythematosus (NPSLE) was made based on history, clinical and investigational findings fulfilling the ACR/EULAR criteria and patient was administered steroids and hydroxychloroquine. His symptoms subsided and on further follow up his systemic lupus erythematosus was quiescent.

\section{Discussion}

Systemic lupus erythematosus being a great imitator has a variety of clinical manifestations and can affect multiple organ systems. Involvement of the nervous system, either primary or secondary, is common in $\mathrm{SLE}^{(5)}$. Several pathophysiological mechanisms have been implicated in the pathogenesis of neuropsychiatric SLE (NPSLE), including vasculopathy, autoantibodies, and cytokine-mediated tissue injury (Table1). However due to limited number of case reports of male patients with systemic lupus erythematosus and patients with neuropsychiatric symptoms as first manifestation of SLE the diagnosis is often delayed and even missed. In this case report we described a 26 year old male patient with neuropsychiatric symptoms as the first manifestation of systemic lupus erythematosus which may help the cause.

Table 1 Pathogenic mechanisms in neuropsychiatric systemic lupus erythematosus

\begin{tabular}{|l|l|}
\hline Vasculopathy & $\begin{array}{l}\text { Small vessel non-inflammatory } \\
\text { vasculopathy } \\
\text { Vascuilitis (rare) }\end{array}$ \\
\hline Autoantibodies & $\begin{array}{l}\text { Procoagulant effect } \\
\text { Direct cytotoxic effect }\end{array}$ \\
\hline Cytokines & $\begin{array}{l}\text { Promotion of antibodies production } \\
\text { Recruitment of immune cells } \\
\text { Alteration of blood brain barrier }\end{array}$ \\
\hline
\end{tabular}

\section{Conclusion}

This case report illustrates the importance of considering the diagnosis of Neuropsychiatric systemic lupus erythematosus in male patients who present initially with psychiatric disorders like psychosis, mood disorders, personality or behavioural changes. Early diagnosis and appropriate treatment may lead to decreased morbidity and mortality in these patients.

\section{References}

1. Brey RL. Neuropsychiatric Lupus, clinical and imaging aspects. Bullet NYU Hosp joint dis. 2007;65(3):194-9. [PubMed] [Google Scholar]

2. $\mathrm{Yu} \mathrm{CH}$, Lee MB, Tseng MM, Liao SC. Obsessive-Compulsive symptoms as a manifestation of neuropsychiatric SLE. J Formus Med Assoc. 2008;107:68-72. [PubMed] [Google Scholar]

3. Harel L, Sandborg Ch, Lee T, Scheven E. Neuropsychiatric manifestations in paediatric SLE and association with antiphospholipid antibodies. J Rheumatol. 2006;33:1873-7. [PubMed] [Google Scholar]

4. Danchenko, N., Satia J., and Anthony M.. 2006. Epidemiology of systemic lupus erythematosus: a comparison of worldwide disease burden. Lupus 15:308-318. [PubMed] [Google Scholar]

5. Ellis SG, Verity MA. Central nervous system involvement in systemic lupus erythematosus: A review of neuropathologic findings in 57 cases, 1956-1977. Semin Arthritis Rheum. 1979;8:212-21. [PubMed] [Google Scholar]. 